\title{
Late-onset Transthyretin (TTR)-familial Amyloid Polyneuropathy (FAP) with a Long Disease Duration from Non-endemic Areas in Japan
}

\author{
Zenshi Miyake ${ }^{1}$, Kiyotaka Nakamagoe ${ }^{2}$, Naoki Ezawa ${ }^{3}$, Tsuneaki Yoshinaga ${ }^{3}$, \\ Ryosuke Hashimoto ${ }^{4}$, Taiki Sato ${ }^{5}$, Yoshiki Sekijima ${ }^{3}$ and Akira Tamaoka ${ }^{2}$
}

\begin{abstract}
:
We herein report the case of an 84-year-old woman with transthyretin (TTR) Val30Met-associated familial amyloid polyneuropathy (FAP-ATTR Val30Met), representing a very old case. The patient had muscle weakness and sensory disturbances in her extremities caused by severe peripheral neuropathy. She also had vitreous opacity and orthostatic hypotension, and pyrophosphate scintigraphy showed a myocardial accumulation. Esophagogastroduodenoscopy revealed mucosal amyloid deposits, positive in anti-TTR antibody staining. A TTR gene analysis isolated the Val30Met mutation. More than a few cases of FAP-ATTR develop late, like our own, and their familial histories are often obscure in non-endemic areas, which might make a diagnosis difficult.
\end{abstract}

Key words: transthyretin familial amyloid polyneuropathy, non-endemic area, Val30Met mutation, polyneuropathy, vitreous opacity, orthostatic hypotension

(Intern Med 58: 713-718, 2019)

(DOI: 10.2169/internalmedicine.1457-18)

\section{Introduction}

In patients with familial amyloid polyneuropathy (FAP), amyloid depositions are seen in the peripheral and autonomic nerves in the early stage. They are seen in multiple organs, such as the heart, gastrointestinal tract, and kidneys in the advanced stage, which impairs the function of organs. Mutant transthyretin, mutant apolipoprotein A1, mutant gelsolin, and mutant beta- 2 microglobulin are known as proteins accumulated in FAP (1-3). In Japan, most patients with FAP are diagnosed with transthyretin-related familial amyloid polyneuropathy (FAP-ATTR). FAP-ATTR shows autosomal dominant inheritance, and several families have been identified and reported. However, there have also been sporadic cases where the familial histories are unclear. While Kumamoto, Nagano, and Ishikawa Prefectures are known as endemic areas, several cases of FAP-ATTR in non-endemic areas have also been reported in Japan (4-6).

We herein report an elderly case of FAP-ATTR in a nonendemic area.

\section{Case Report}

An 84-year-old woman visited our hospital in a wheel chair with complaints of muscle weakness and sensory disturbances in the extremities. She had a history of hypertension and bradyarrhythmia but no family history of cardiac or peripheral nerve diseases. The patient had no history of smoking or drinking. She had begun to experience orthostatic dizziness in her 50s. At 78 years of age, she noticed left impairment of visual acuity, and an ophthalmologic examination showed left vitreous opacity and bilateral cataracts. Around 81 years of age, she began to experience tingling sensations in her fingers and toes. One year later, she became short of breath and sometimes experienced diz-

\footnotetext{
${ }^{1}$ Department of Neurology, University of Tsukuba Hospital, Japan, ${ }^{2}$ Department of Neurology, Faculty of Medicine, University of Tsukuba, Japan, ${ }^{3}$ Department of Medicine (Neurology and Rheumatology), Shinshu University School of Medicine, Japan, ${ }^{4}$ Department of Pathology, University of Tsukuba Hospital, Japan and ${ }^{5}$ Department of Diagnostic Pathology, Faculty of Medicine, University of Tsukuba, Japan Received: April 27, 2018; Accepted: July 22, 2018; Advance Publication by J-STAGE: October 17, 2018 Correspondence to Dr. Kiyotaka Nakamagoe, Nakamagoek@md.tsukuba.ac.jp
} 
A

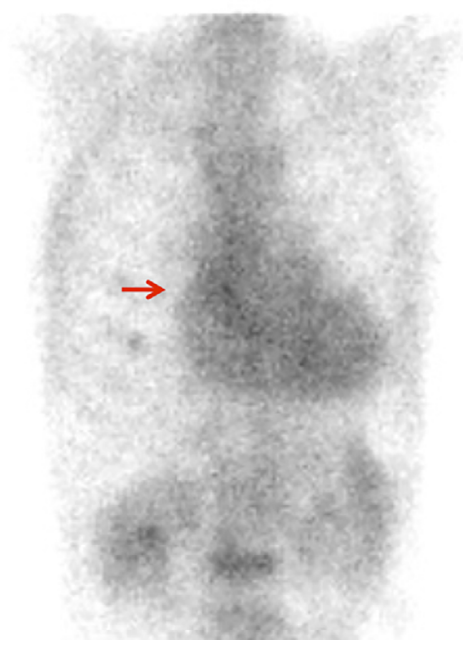

B

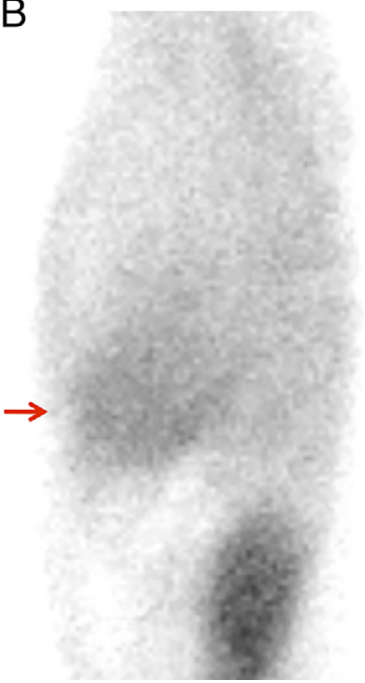

Figure 1. Technetium-99m-pyrophosphate scintigraphy. An intense myocardial uptake compared with that of bones was found (red arrows).

ziness. She lost her appetite due to nausea. At 83 years of age, she developed distal muscle weakness in her extremities, which led to difficulty writing and opening caps of PET bottles. She also needed support to walk. She was admitted to our hospital for an examination and treatment.

On admission, she had a blood pressure of 100/52 $\mathrm{mmHg}$ (126/63 mmHg on average during hospitalization), pulse rate of $64 / \mathrm{min}$ (regularly irregular), body temperature of $36.4{ }^{\circ} \mathrm{C}$, percutaneous oxygen saturation of $99 \%$, and a height and weight of $147.6 \mathrm{~cm}$ and $33.6 \mathrm{~kg}$, respectively (body mass index: $15.4 \mathrm{~kg} / \mathrm{m}^{3}$ ). The third heart sound was heard with auscultation. During a neurological examination, she was alert and conscious. She had no abnormality in the cranial nervous system except for hearing loss on the right side. Muscle weakness was noted predominantly in the distal extremities, and muscle atrophy was found in the bilateral interosseous dorsalis muscles, balls of the thumbs, hypothenar areas, and tibialis anterior muscles. Deep tendon reflexes were absent in all limbs, and no pathological reflexes were observed. She had tactile and pain anesthesia in all distal portions of her extremities and decreased vibration and position sensation in all limbs. She also had dysesthesia with a glove-stocking distribution. No cerebellar ataxia was noted. She had hypohidrosis, nocturia, and recurrent diarrhea and constipation. An ophthalmologic examination at our hospital revealed vitreous opacity.

The complete blood cell count values were white blood cells $3,300 / \mu \mathrm{L}$, red blood cells 3.54 million $/ \mu \mathrm{L}$, hemoglobin $10.6 \mathrm{~g} / \mathrm{dL}$, hematocrit $33.0 \%$, platelets $156,000 / \mu \mathrm{L}$, and erythrocyte sedimentation rate $12 \mathrm{~mm} / \mathrm{h}$. The coagulation and fibrinolytic systems were normal. The patient's Nterminal pro-brain natriuretic peptide was $686.70 \mathrm{pg} / \mathrm{dL}$ (normal range $<125 \mathrm{pg} / \mathrm{dL}$ ), and both immunoglobulin A and $\mathrm{M}$ were below the lower limit $(109 \mathrm{~m} / \mathrm{dL}$ and $30 \mathrm{mg} /$ dL, respectively ). Angiotensin-converting enzyme, myeloperoxidase-anti-neutrophil cytoplasmic antibody, and proteinase 3-anti-neutrophil cytoplasmic antibody levels were normal, and anti-glycolipid antibodies were all negative. Neither serum M protein nor urinary Bence Jones Protein was detected. A cerebrospinal fluid analysis revealed normocytosis with an increased protein concentration of 51 $\mathrm{mg} / \mathrm{dL}$ (normal range $<40 \mathrm{mg} / \mathrm{dL}$ ).

On an electrocardiogram, a normal sinus rhythm was noted, but an rS pattern in lead II, a QS pattern in leads III and $\mathrm{aV}_{\mathrm{F}}$, and a low voltage in all leads were found. Holter electrocardiography revealed sporadic premature ventricular contractions and no atrioventricular block. Transthoracic cardiac ultrasonography showed an ejection fraction of $65 \%$ (modified Simpson's method), intervascular septum thickness of $12.6 \mathrm{~mm}$, left ventricular posterior wall thickness of $12.2 \mathrm{~mm}$, relative wall thickness at the end of diastole of 0.64 , and ratio of the early diastolic transmitral flow velocity to the mitral annular velocity (e/e') of 13.7, which suggested prominent left centripetal ventricular hypertrophy and diastolic dysfunction. While thallium myocardial scintigraphy revealed no accumulation in cardiac tissues, technetium99m-pyrophosphate scintigraphy found an intense myocardial uptake compared with that of bones (Fig. 1). The Schellong test indicated prominent orthostatic hypotension (blood pressure $135 / 54 \mathrm{mmHg}$ while lying down and $83 / 65 \mathrm{mmHg}$ at 1 minute after standing).

Whole-body contrast computed tomography (CT) revealed no malignant tumors. Magnetic resonance imaging (MRI) of the head revealed only chronic ischemic changes. Cervical spine contrast MRI showed bulging of the C4/5 disc and mild stenosis of the spinal cord; no abnormal signals or enhancing effects in the spinal cord or nerve roots were found.

A motor nerve conduction analysis showed prolongation of the distal latency and decreased amplitude of the compound muscle action potential (CMAP) in the median and tibial nerves, and CMAP was not detected in the peroneal nerve. It was also impossible to detect sensory nerve action 
Table 1. Nerve Conduction Study in Right Upper and Lower Limbs.

\begin{tabular}{|c|c|c|c|c|}
\hline Nerve & & & First time & $\begin{array}{l}1 \text { year after } \\
\text { the first time }\end{array}$ \\
\hline \multirow[t]{5}{*}{ Median nerve } & Motor & $\operatorname{MCV}(\mathrm{m} / \mathrm{s})$ & 48.1 & 44.9 \\
\hline & & DL (ms) & 4.6 & 5.0 \\
\hline & & CMAP (mV) & 2.4 & 2.8 \\
\hline & Sensory & $\mathrm{SCV}(\mathrm{m} / \mathrm{s})$ & $\mathrm{NE}$ & $\mathrm{NE}$ \\
\hline & & $\operatorname{SNAP}(\mu \mathrm{V})$ & & \\
\hline \multirow[t]{5}{*}{ Ulnar nerve } & Motor & $\operatorname{MCV}(\mathrm{m} / \mathrm{s})$ & 59.7 & 55.6 \\
\hline & & DL (ms) & 2.9 & 2.8 \\
\hline & & CMAP (mV) & 8.4 & 6.2 \\
\hline & Sensory & $\mathrm{SCV}(\mathrm{m} / \mathrm{s})$ & $\mathrm{NE}$ & $\mathrm{NE}$ \\
\hline & & $\operatorname{SNAP}(\mu \mathrm{V})$ & & \\
\hline \multirow[t]{3}{*}{ Tibial nerve } & Motor & $\operatorname{MCV}(\mathrm{m} / \mathrm{s})$ & 25.5 & $\mathrm{NE}$ \\
\hline & & DL (ms) & 11.6 & \\
\hline & & CMAP (mV) & 0.32 & \\
\hline \multirow[t]{2}{*}{ Sural nerve } & Sensory & $\mathrm{SCV}(\mathrm{m} / \mathrm{s})$ & $\mathrm{NE}$ & $\mathrm{NE}$ \\
\hline & & $\operatorname{SNAP}(\mu \mathrm{V})$ & & \\
\hline
\end{tabular}

MCV: motor nerve conduction velocity, DL: distal latency, CMAP: compound muscle action potentials, SCV: sensory nerve conduction velocity, SNAP: sensory nerve action potentials, NE: not elicited

potential (SNAP) in the median, ulnar, and sural nerves (Table 1). These results suggested mixed-type (predominantly axonal) peripheral neuropathy with carpal tunnel syndrome. Needle electromyography in the right biceps brachii and tibialis anterior muscles revealed polyphasic and motor unit potentials (MUPs) as well as high-amplitude MUPs, indicating chronic denervation.

Based on the results of the Schellong test and myocardial pyrophosphate scintigraphy, amyloid polyneuropathy was suspected as the cause of her severe peripheral neuropathy. A gastroduodenal biopsy with esophagogastroduodenoscopy and sural nerve biopsy were therefore performed. The gastroduodenal biopsy revealed infiltration of lymphocytes, plasma cells, and eosinophils into the stroma, and amyloid deposits with Congo Red staining, which were specifically stained with anti-transthyretin ( TTR ) antibodies (Fig. 2A and B). The sural nerve biopsy revealed a mild decrease in the amount of myelinated nerves; however, no significant demyelination or inflammation was noted. Amyloid deposits that were positive on anti-TTR antibody staining were noted in the vicinity of the nerve bundles in the perineurium (Fig. 2C and D).

Matrix-assisted laser desorption ionization time-of-flight mass spectrometry (MALDI-TOF-MS) revealed the peak of mutant TTR, whose molecular weight was lower than that of wild-type TTR by 30, in addition to the peak of wildtype TTR, which indicated a Val30Met mutation. A TTR gene analysis was performed after the patient gave informed consent. We finally diagnosed the patient with FAP-ATTR (Val30Met) (Fig. 3).

Before the results of a gastroduodenal and sural nerve biopsy were determined, we tentatively diagnosed her with an immune-mediated neuropathy and performed intravenous immunoglobulin (IVIg) therapy, with no improvement. She started taking tafamidis after her diagnosis. Her miscellaneous hand activities, such as buttoning and unbuttoning, were partially improved. One year after the administration of tafamidis, a nerve conduction study revealed no remarkable changes in the upper limbs (Table 1).

\section{Discussion}

We herein report a very old case of FAP. Amyloid deposits are found in the peripheral and autonomic nerves of patients with early-stage FAP. The main symptoms of FAP are polyneuropathy and autonomic dysfunction. Deposited amyloid proteins include mutant TTR, mutant apolipoprotein AI, mutant gelsolin, and mutant beta-2 microglobulin (1-3). Most cases of FAP in Japan are classified as FAP-ATTRinherited as an autosomal dominant trait. Kumamoto, Nagano, and Ishikawa Prefectures are known as endemic areas; however, several sporadic cases have also been reported in other areas. These sporadic cases are marked by an advanced age at onset, slowly progressive polyneuropathy, and cardiac lesions $(1,4)$. Amyloids identified in Japan contain one amino acid replacement of TTR, often Val30Met. Cases of FAP-ATTR in non-endemic areas, such as the present one, often have no familial history (Table 2) $(7,8)$.

In cases of early onset FAP-ATTR Val30Met, sensory disturbances in the lower extremities due to polyneuropathy, diarrhea and constipation because of dysautonomia, digestive symptoms such as nausea and vomiting, syncope caused by orthostatic hypotension, and erectile dysfunction are seen in the early stage, and atrioventricular blocks are often noted (1). However, in cases of elderly-onset FAP-ATTR Val30Met, sensory disturbance in the lower limbs is marked, 


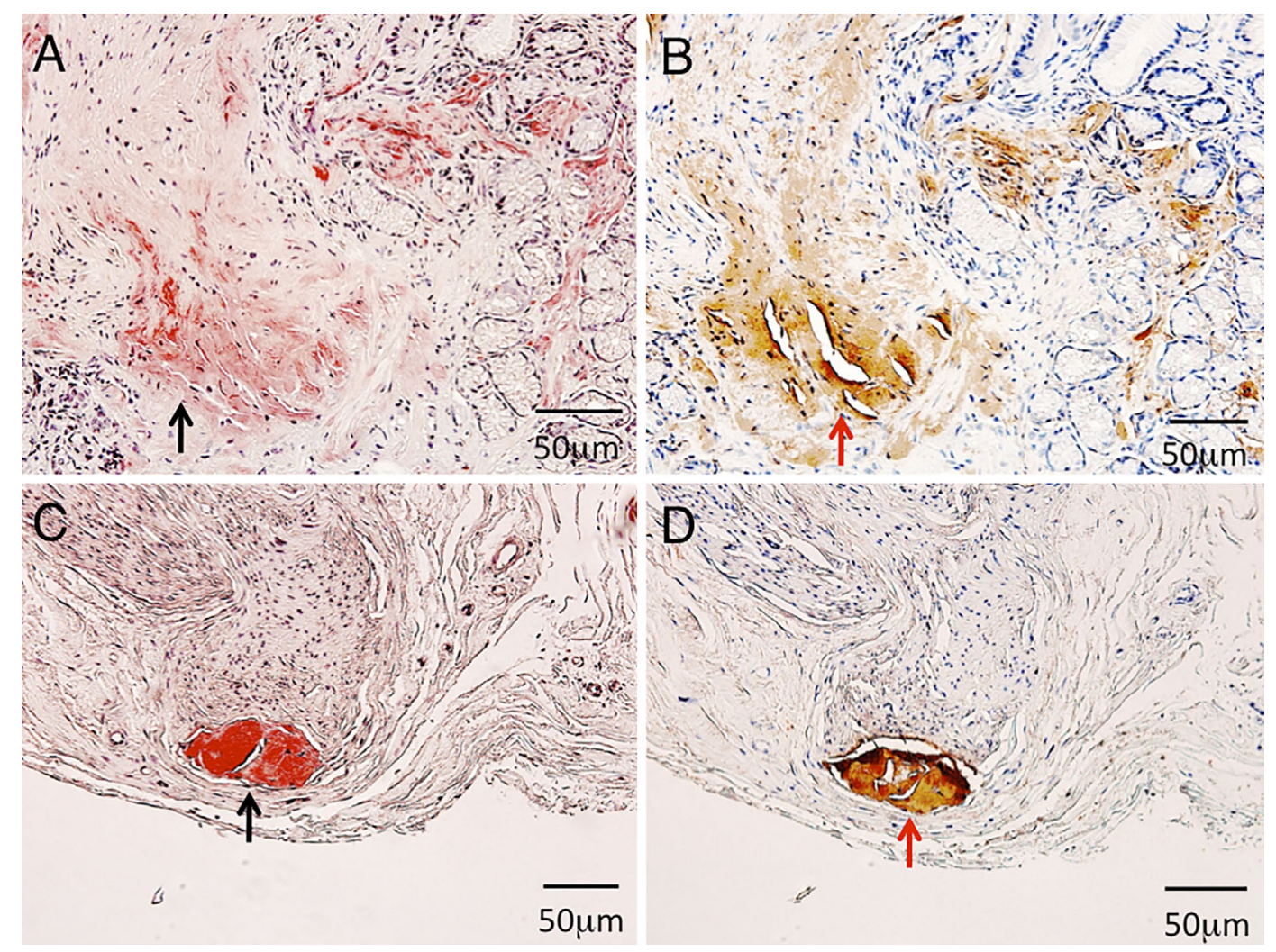

Figure 2. Biopsies of the gastric mucosa (A, B) and sural nerve (C, D). A: Congo Red staining revealed amyloid deposits in the muscularis mucosa and submucosa (black arrow). B: The amyloid deposits shown in (A) specifically stained with anti-transthyretin (TTR) antibodies (red arrow). C: Congo Red staining revealed amyloid deposits under the perineurium (black arrow). D: The amyloid deposits shown in (C) were specifically stained with anti-TTR antibodies (red arrow).

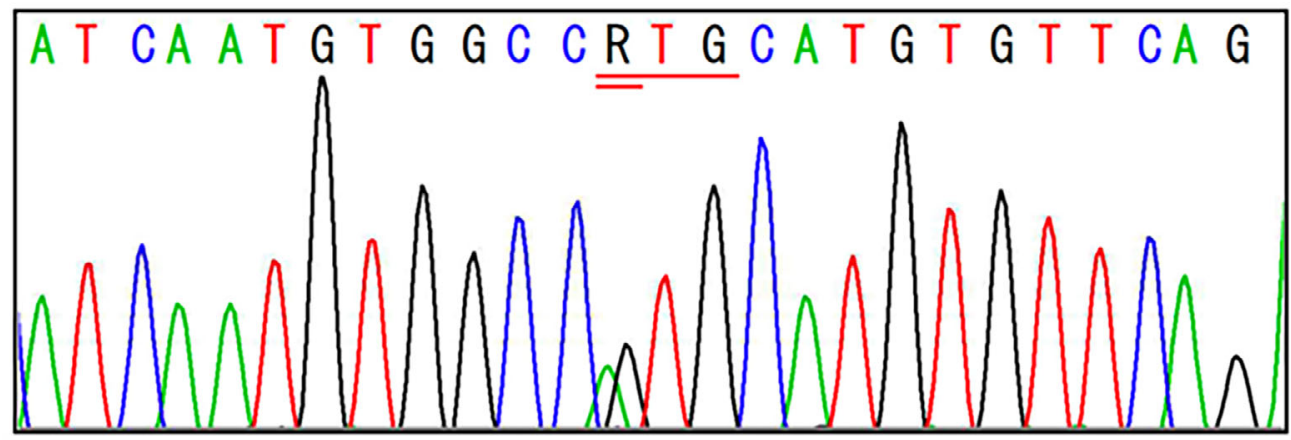

\section{Reading sequence ATCAATGTGGCCRTGCATGTGTTCA \\ RefSeq sequence A T CAATGTGGCCGTGCATGTGTTCA G \\ Mismatched sequence A T CA A T T G C CATGCATGTGTTCA G}

\section{RefSeq; Reference Sequence Database}

Figure 3. An analysis of the TTR gene. A Val30Met (V30M) mutation was identified.

while autonomic dysfunction is mild in the initial stage, and cardiac hypertrophy is frequently found $(4,6)$.

The vitreous opacity seen in the present case, caused by the accumulation of amyloids, is often found in patients with FAP-ATTR. This symptom may help with the diagnosis (9).

In the present case, the patient manifested slowly progressive muscle weakness and sensory disturbances in the ex- 
Table 2. Comparison of Characteristics of TTR-FAP (Val30Met) in Japan with Our Case (12).

\begin{tabular}{lccc}
\hline & $\begin{array}{c}\text { Early-onset cases } \\
\text { from endemic areas }\end{array}$ & $\begin{array}{c}\text { Late-onset cases } \\
\text { from non-endemic areas }\end{array}$ \\
\hline Age at onset (years) & $34.4 \pm 6.4$ & $64.0 \pm 6.4$ \\
Men/women & $0.9 / 1$ & $4.5 / 1$ & Rare case \\
Presence of family history & Common & Somsory deficit & Rone \\
Initial symptom of neuropathy & Autonomic dysfunction & Autonomic dysfunction \\
Sensory dissociation & Common & None \\
Autonomic dysfunction in early stages & Severe with ADL disturbances & Mild without ADL disturbance & Moderate \\
Cardiac involvement & Atrioventricular conduction block & Cardiomegaly \\
\hline
\end{tabular}

TTR: transthyretin, ADL: activity of daily life

tremities. A neurological examination revealed distaldominant muscle weakness and decreased superficial and deep sensations that showed a glove-and-stocking distribution. A nerve conduction study suggested mixed peripheral neuropathy-mainly axonopathy. Focusing only on the progressive motor and sensory polyneuropathy made us suspect the possibility of an immune-mediated neuropathy, such as acute motor and sensory axonal neuropathy. Thus, IVIg therapy was performed, with no improvement. We also noted carpal tunnel syndrome, which has been reported as an initial symptom of FAP-ATTR (10).

The present patient developed orthostatic dizziness in her 50s. If we consider such dizziness the initial symptom of FAP-ATTR in this patient, it follows that she suffered from the disease for about 30 years, which is much longer than the natural history of elderly-onset FAP-ATTR in nonendemic areas (11). It is possible that the dizziness resulted from orthostatic hypotension due to cardiovascular autonomic impairment. However, the results of cardiac ultrasonography and technetium-99m-pyrophosphate scintigraphy suggested the presence of cardiac amyloidosis, so it is also possible that the orthostatic dizziness was a symptom of cardiac amyloidosis through diastolic dysfunction or arrhythmia independent of dysfunction of the autonomic nervous system (12).

More than $95 \%$ of TTR is produced in the liver, so liver transplantation from a living donor has been established as the treatment of FAP-ATTR for the purpose of suppressing the production of the mutant TTR. Nonetheless, about $80 \%$ of patients are actually inoperable because of their age, disease progression, or other factors (13). Both normal and mutant TTR proteins are known to form tetramers. Diflunisal and tafamidis have both been reported to delay the progression of neuropathy by preventing the dissociation of unstable tetramers composed of mutant TTR, leading to a decreased amyloid production $(14,15)$. In the present case, the administration of tafamidis mildly improved muscle weakness and dysesthesia in the limbs.

For cases of FAP-ATTR in non-endemic areas, such as our own, a diagnosis is often difficult and takes a long time. If patients have any autonomic dysfunctions and/or defects in the eyes or heart in addition to sensory and motor neuropathy, FAP should be considered as a differential diagnosis, and tissue biopsies from gastric mucosa and subcutaneous fat should be positively examined. It is important to diagnose FAP, since some drugs can prevent the progression of the disease.

The authors state that they have no Conflict of Interest (COI).

\section{Acknowledgement}

We thank Dr. A. Ishii (Department of Neurology, Faculty of Medicine, University of Tsukuba), for obtaining the findings of the nerve biopsy of the patient.

\section{References}

1. Ando Y. Amyloidosis and neurological disorders: treatable amyloidosis. Rinsho Shinkeigaku 55: 797-803, 2015 (in Japanese, Abstract in English).

2. Westermark P, Benson MD, Buxbaum JN, et al. A primer of amyloid nomenclature. Amyloid 14: 179-183, 2007.

3. Ando Y, Coelho T, Berk JL, et al. Guideline of transthyretinrelated hereditary amyloidosis for clinicians. Orphanet $\mathrm{J}$ Rare Dis 8: 31-48, 2013.

4. Koike H, Sobue G. Late-onset familial amyloid polyneuropathy in Japan. Amyloid 19: 55-57, 2012.

5. Koike H, Nakamura T, Hashizume A, et al. Cardiac and peripheral vasomotor autonomic functions in late-onset transthyretin Val30Met familial amyloid polyneuropathy. J Neurol 264: 22932302, 2017.

6. Sato C, Takaya T, Mori S, et al. An isolated case of late-onset amyloidogenic transthyretin type familial amyloid polyneuropathy associated with a mutant transthyretin substituting methionine for valine at position 30 showing latent progressive cardiac involvement confirmed by serial annual electrocardiograms. Intern Med 56: 163-168, 2017.

7. Koike H, Sobue G. Clinicopathological features of familial amyloid polyneuropathy. Rinsho Shinkeigaku 51: 1134-1137, 2011.

8. Koike H, Hashimoto R, Tomita M, et al. Diagnosis of sporadic transthyretin Val30Met familial amyloid polyneuropathy: a practical analysis. Amyloid 18: 53-62, 2011.

9. Kawaji T, Ando Y, Ando E, et al. Transthyretin-related vitreous amyloidosis in different endemic areas. Amyloid 17: 105-108, 2010.

10. Tojo K, Tsuchiya-Suzuki A, Sekijima Y, et al. Upper limb neuropathy such as carpal tunnel syndrome as an initial manifestation of ATTR Val30Met familial amyloid polyneuropathy. Amyloid 17: 32-35, 2010.

11. Koike H, Tanaka F, Hashimoto R, et al. Natural history of transthyretin Val30Met familial amyloid polyneuropathy: analysis of late-onset cases from non-endemic areas. J Neurol Neurosurg 
Psychiatry 83: 152-158, 2012.

12. Mankad AK, Shah KB. Transthyretin cardiac amyloidosis. Curr Cardiol Rep 19: 97-107, 2017.

13. Sekijima Y. New treatment for familial amyloid polyneuropathy. Shinshu Igaku Zasshi 62: 185-187, 2014 (in Japanese, Abstract in English).

14. Sekijima Y, Tojo K, Morita H, et al. Safety and efficacy of longterm diflunisal administration in hereditary transthyretin (ATTR) amyloidosis. Amyloid 22: 79-83, 2015.
15. Coelho T, Maia LF, Martins da Silva A, et al. Tafamidis for transthyretin familial amyloid polyneuropathy: a randomized, controlled trial. Neurology 79: 785-792, 2012.

The Internal Medicine is an Open Access journal distributed under the Creative Commons Attribution-NonCommercial-NoDerivatives 4.0 International License. To view the details of this license, please visit (https://creativecommons.org/licenses/ by-nc-nd/4.0/).

(C) 2019 The Japanese Society of Internal Medicine Intern Med 58: 713-718, 2019 